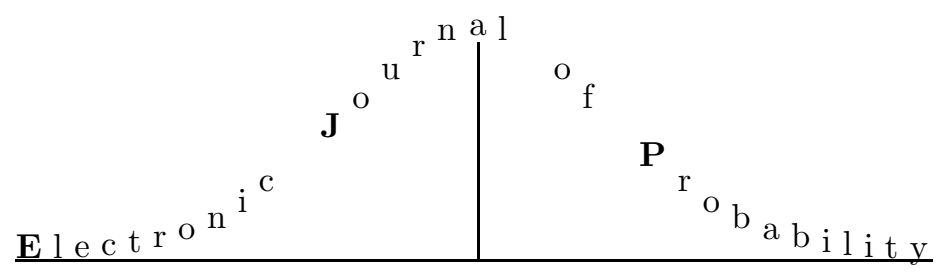

Vol. 12 (2007), Paper no. 30, pages 862-887.

Journal URL

http://www.math. washington.edu/ ejpecp/

\title{
Number variance from a probabilistic perspective: infinite systems of independent Brownian motions and symmetric $\alpha$-stable processes
}

\author{
Ben Hambly and Liza Jones* \\ Mathematical Institute, \\ University of Oxford, \\ 24-29 St Giles', \\ Oxford, \\ OX1 3LB.
}

hambly@maths.ox.ac.uk, jones@maths.ox.ac.uk

\begin{abstract}
Some probabilistic aspects of the number variance statistic are investigated. Infinite systems of independent Brownian motions and symmetric $\alpha$-stable processes are used to construct explicit new examples of processes which exhibit both divergent and saturating number variance behaviour. We derive a general expression for the number variance for the spatial particle configurations arising from these systems and this enables us to deduce various limiting distribution results for the fluctuations of the associated counting functions. In particular, knowledge of the number variance allows us to introduce and characterize a novel family of centered, long memory Gaussian processes. We obtain fractional Brownian motion as a weak limit of these constructed processes.
\end{abstract}

Key words: Number variance, symmetric $\alpha$-stable processes, controlled variability, Gaussian fluctuations, functional limits, long memory, Gaussian processes, fractional Brownian motion.

\footnotetext{
${ }^{*}$ Research supported by the EPSRC through the Doctoral Training Account scheme
} 
AMS 2000 Subject Classification: Primary 60G52, 60G15; Secondary:60F17, 15A52.

Submitted to EJP on July 14, 2006, final version accepted June 13, 2007. 


\section{Introduction}

Let $(X, \mathcal{F}, \mathbb{P})$ be a point process on $\mathbb{R}$, that is, a collection $X:=\left\{\left(x_{i}\right)_{i=-\infty}^{\infty}: x_{i} \in\right.$ $\mathbb{R}, \forall i$ and $\left.\#\left(x_{i}: x_{i} \in[b, b+L]\right)<\infty \forall b \in \mathbb{R}, L \in \mathbb{R}_{+}\right\}$, with $\mathcal{F}$ the minimal $\sigma$-algebra generated by these point configurations and $\mathbb{P}$ some probability measure on $(X, \mathcal{F})$. The associated counting function is

$$
N[b, b+L]=\#\left(x_{i}: x_{i} \in[b, b+L]\right)
$$

The number variance is then defined as

$$
\operatorname{Var}_{\mathbb{P}}(N[b, b+L])
$$

More generally, in order to deal with non-spatially homogeneous cases, it is more convenient to work with the averaged number variance which we define as

$$
V[L]:=\mathbb{E}_{b}[\operatorname{Var}(N[b, b+L])]
$$

taking an appropriate uniform average of the number variance over different intervals of the same length. As we increase the length $L$ of the underlying interval the number of points contained in that interval will also increase. However, in many situations, it is not immediately clear what will happen to the variance of this number as the interval length grows. One of the main questions considered in this paper will be the behaviour of $V[L]$ as $L \rightarrow \infty$. We shall see that, somewhat counter-intuitively, in some instances we have

$$
\lim _{L \rightarrow \infty} V[L]=\kappa<\infty
$$

in which case we will say that the number variance saturates to the level $\kappa \in \mathbb{R}_{+}$.

The number variance statistic arises in a wide variety of contexts; effectively in any situation in which points or occurrences of events are counted. In many of these cases, it is advantageous to determine the growth behaviour of the statistic. In the fields of random matrix theory (see e.g. $(22 ; 13))$ and quantum theory $($ e.g $(3 ; 20)$ ) for instance this is used as an indicator of spectral rigidity. In the study of quantum spectra, the manner in which the number variance (of eigenvalues) grows with interval length provides an indication of whether the underlying classical trajectories are integrable, chaotic or mixed. In the large energy limit, the spectral statistics of a quantum system with strongly chaotic classical dynamics, should, according to the conjecture of Bohigas, Giannoni and Schmidt (7), agree with the corresponding random matrix statistics which are independent of the specific system. However, in reality, for many such systems, when it comes to the long range "global" statistics, this random matrix universality breaks down. In these cases, the number variance typifies this transition to non-conformity in that following an initial random matrix consistent logarithmic growth with interval length, the number variance then saturates.

Attempts to improve the understanding of the deviations from random matrix predictions have led to convincing explanations for number variance saturation behaviour in terms of periodic orbit theory, see for example (4; 5 ; 1 ). In (4) there is a heuristic derivation of an explicit formula for the empirical number variance of the zeros of the Riemann zeta function on the critical line which is consistent with numerical evidence.

Asymptotically bounded and saturating number variances have also been studied in the literature on point processes where the property is referred to as that of "controlled variability". See for 
instance $(14 ; 19)$ and the more recent article (11) which provide interesting examples with applications to the outputs of certain queues.

In the last few years, number variance has been considered from a slightly different viewpoint in relation to point processes with determinantal structure (see (27) for background on this topic). Results on the growth of the number variance for these processes are given in e.g. (27; 28). The emphasis in these cases is on ascertaining the divergence of the number variance as this, it turns out, is the key ingredient needed to prove Gaussian fluctuation results for the counting functions of a large class of determinantal processes, including those arising naturally in the context of random matrix theory.

Motivated by the Riemann zeta example, Johansson (16) recently introduced an example of a point process with determinantal structure which demonstrates the same type of number variance saturation behaviour as conjectured by Berry $((4))$ for the Riemann zeroes. This process is constructed from a system of $n$ non-colliding Brownian particles started from equidistant points $u_{j}=\Upsilon+a(n-j)$ with $\Upsilon \in \mathbb{R}, a \in \mathbb{R}_{+} \quad j=1, \ldots, n$. There are a number of approaches to describing such a system, see $(15 ; 17)$ for details. In any case, it can be shown that the configuration of particles in space formed by the process at a fixed time $t$, is a determinantal process and as such its correlation functions (or joint intensities) $R_{m}^{(n)}$ take the form

$$
R_{m}^{(n)}\left(x_{1}, x_{2}, \ldots, x_{m}\right) \mathrm{d} x_{1} \ldots \mathrm{d} x_{m}=\operatorname{det}\left(K_{t}^{(n)}\left(x_{i}, x_{j}\right)\right)_{i, j=1}^{m} \mathrm{~d} x_{1} \ldots \mathrm{d} x_{m} .
$$

Here and for determinantal processes in general, the above expression may be interpreted as the probability of finding $m$ of the points in the infinitesimal intervals around $x_{1}, x_{2}, \ldots, x_{m}$. The exact expression for the correlation kernel $K_{t}^{(n)}$ can be found in (16)

As the number of particles $n \rightarrow \infty$, Johansson shows that the correlation kernel $K_{t}^{(n)}$ converges uniformly to a limiting kernel which defines, at each time $t$, a limiting, non-spatially homogeneous determinantal process. The resulting process may loosely be thought of as the fixed time spatial particle configuration formed by an infinite system of non-colliding Brownian particles started from an equispaced initial configuration. When the interval length $L$ is small relative to $d:=\frac{2 \pi t}{a^{2}}$, the averaged number variance for this process has leading term

$$
\frac{1}{\pi^{2}}\left(\log (2 \pi L / a)+\gamma_{\text {Euler }}+1\right) .
$$

However, if $d$ is held constant, while $L$ is increased, it is deduced that the number variance saturates to the level

$$
\frac{1}{\pi^{2}}\left(\log (2 \pi d)+\gamma_{\text {Euler }}+1\right) .
$$

The "small L" expression (1.2) agrees with the number variance of the determinantal point process associated with the sine kernel of density $a$, which is the universal scaling limit obtained for the eigenvalues of random matrices from e.g. the Gaussian Unitary Ensemble and U(n), as matrix size tends to infinity (see for example, (22)).

For our purposes it will be convenient to think of the above averaged number variance as the number variance of the spatial particle configurations arising from an "averaged model" which we choose to interpret as an infinite system of non-colliding Brownian motions started from the initial positions

$$
u_{j}=a(j-\epsilon), \quad j \in \mathbb{Z}, \quad a \in \mathbb{R}_{+}, \quad \epsilon \sim \operatorname{Uniform}[0,1] .
$$


In this work we consider the number variance statistic in the independent process analogues of Johansson's model. Since these independent versions do not fall into the previously considered frameworks mentioned above, existing number variance results no longer apply.

The paper is organized as follows. We begin by deriving an explicit general formula for the number variance for the spatial particle configurations arising from infinite systems of independent Brownian motions and symmetric $\alpha$-stable processes in $\mathbb{R}$ started from the initial configuration (1.4). This enables us to deduce the asymptotic behaviour of the statistic as the length of the interval over which it is defined goes to infinity. We give a precise formula for the saturation level for the cases in which saturation does occur. Once this is achieved we are able to explain the number variance saturation phenomenon in terms of the tail distribution behaviours of the underlying processes. We provide two specific illustrative examples as corollaries. We conclude the first section by demonstrating the close relationship between the number variance and the rate of convergence of the distribution of the associated counting function to a Poisson law.

In the second section we use the number variance to prove Gaussian fluctuation results for the counting functions of our particle configurations in two different scalings. In the third and final section we add some dynamics to the fluctuations of the counting functions to construct a collection of processes, each of which is shown to converge weakly in $C[0, \infty)$ to a centered Gaussian process with covariance structure similar in form to that of a fractional Brownian motion. Our earlier results on the behaviour of the number variance allow us to better characterize these limiting processes. In particular, the long-range dependence property exhibited by the covariance of their increments is directly determined by the rate of growth of the associated number variance. In the cases corresponding to $\alpha \in(0,1)$, a further rescaling of the limiting Gaussian processes allow us to recover fractional Brownian motions of Hurst parameters $\frac{1-\alpha}{2}$ as weak limits.

\section{The independent particle cases}

\subsection{A Poisson process initial configuration}

We begin by illustrating the effect of the initial positions on the number variance of the spatial particle configurations arising from such infinite systems of processes as those considered in this paper. The following theorem is the well known result (see for example, (9), Chapter VIII, section 5) that the Poisson process is invariant for an infinite system of independent particles with the same evolution.

Theorem 2.1. Consider an infinite collection of independent, identical in law, translation invariant real-valued stochastic processes $\left\{\left(X_{j}(t), t \geq 0\right) ; j \in \mathbb{Z}\right\}$. Suppose that $\left\{X_{j}(0)\right\}_{j=-\infty}^{\infty}$ is a Poisson process of intensity $\theta$ on $\mathbb{R}$. Then $\left\{X_{j}(t)\right\}_{j=-\infty}^{\infty}$ is a Poisson process of intensity $\theta$ for every $t$.

Consequently, if we begin with a "mixed up" Poisson process initial configuration and allow each particle to move as (say) a Lévy process, independently of the others then we observe a non-saturating, linearly growing number variance $(V[L]=\theta L)$ at the start and for all time. 


\subsection{The Brownian and symmetric $\alpha$-stable cases}

This last theorem served to highlight the importance of the regularity or rigidity of the starting configuration (1.4) in determining the number variance behaviour. However, it is reasonable to suppose, that in Johansson's model, the strong restrictions placed on the movement of the Brownian particles must also contribute significantly to the saturation behaviour that its number variance demonstrates. This leads us to ask; what would happen if we started with an initial configuration such as (1.4) but did not place such strong restrictions on the movement of the particles? We answer this question for the cases in which each particle moves independently as a one-dimensional Brownian motion or as a symmetric $\alpha$-stable process on $\mathbb{R}$.

Recall (see e.g. (24)) that a symmetric $\alpha$-stable process is a Lévy process $\left(X^{\alpha, c}(t), t \geq 0\right)$ with characteristic function, for each $t \geq 0$, given by

$$
\begin{aligned}
\phi_{X^{\alpha, c}(t)}(\theta) & :=\mathbb{E}\left[e^{i \theta X^{\alpha, c}(t)}\right] \\
& =\exp \left(-t c|\theta|^{\alpha}\right), \quad c>0, \quad \alpha \in(0,2) .
\end{aligned}
$$

Some of the properties enjoyed by this class of processes are;

- $\left\{X^{\alpha, c}(t), t \geq 0\right\}$ with associated transition density $p_{t}(x, y), x, y \in \mathbb{R}$ is temporally and spatially homogeneous.

- $\left\{X^{\alpha, c}(t)\right\}$ is symmetric and self-similar in the sense that $\left\{X^{\alpha, c}(t)\right\} \stackrel{\text { dist }}{=}\left\{-X^{\alpha, c}(t)\right\}$ and $\left\{\lambda^{-1 / \alpha} X^{\alpha, c}(\lambda t)\right\} \stackrel{\text { dist }}{=}\left\{X^{\alpha, c}(t)\right\}$ for constants $\lambda \in \mathbb{R}$.

The arguments that follow also apply to the $\alpha=2$ Gaussian cases. Note that we have standard Brownian motion when $\alpha=2, c=\frac{1}{2}$.

Theorem 2.2. Fix a symmetric $\alpha$-stable process $\left(X^{\alpha, c}(t), t \geq 0\right)$ on $\mathbb{R}$ with properties as described above. Suppose we start an independent copy of this process from each of the starting positions

$$
u_{j}:=a(j-\epsilon), \quad j \in \mathbb{Z}, \quad \text { where } a \in \mathbb{R}_{+} \text {and } \epsilon \sim \operatorname{Uniform}[0,1] .
$$

The configuration of particles in space formed by this infinite system of independent symmetric $\alpha$-stable processes at a fixed time $t$ has number variance

$$
\begin{aligned}
V_{t}^{\alpha, c, a}[L] & =\frac{L}{a}+\frac{2}{\pi} \int_{0}^{\infty} \frac{e^{-2 c t(\theta / a)^{\alpha}}}{\theta^{2}}\left[\cos \left(\frac{L \theta}{a}\right)-1\right] \mathrm{d} \theta \\
& =\frac{4 L}{a \pi} \int_{0}^{\infty} \frac{\sin ^{2}(u / 2)}{u^{2}}\left(1-e^{-2 c t(u / L)^{\alpha}}\right) \mathrm{d} u .
\end{aligned}
$$

Proof. Let $\left\{\left(X_{j}^{\alpha, c}(t), t \geq 0\right), j \in \mathbb{Z}\right\}$ be the independent copies of the chosen symmetric $\alpha$-stable process indexed by $j \in \mathbb{Z}$. Denote the law of each $X_{j}^{\alpha, c}$ started from $x \in \mathbb{R}$ by $\mathbb{P}^{x}$, and write $\mathbb{P}:=\mathbb{P}^{0}$. Now the number of symmetric $\alpha$-stable particles in an interval $[0, L] \subset \mathbb{R}$ at time $t$ is given by the sum of indicator random variables

$$
N_{t}^{\alpha, c, a}[0, L]=\sum_{j=-\infty}^{\infty} \mathbb{I}\left[X_{j}^{\alpha, c}(t)+u_{j} \in[0, L]\right],
$$


where $\left(u_{j}\right)_{j=-\infty}^{\infty}$ is given by (2.2). Note that by construction, for this "averaged model", for all $b \in \mathbb{R}$ we have $N_{t}^{\alpha, c, a}[0, L] \stackrel{\text { dist }}{=} N_{t}^{\alpha, c, a}[b, b+L]$. Thus the number variance is given by

$$
\begin{aligned}
V_{t}^{\alpha, c, a}[L] & :=\operatorname{Var}\left[N_{t}^{\alpha, c, a}[0, L]\right] \\
& =\sum_{j=-\infty}^{\infty} \mathbb{P}\left[X_{j}^{\alpha, c}(t)+u_{j} \in[0, L]\right] \mathbb{P}\left[X_{j}^{\alpha, c}(t)+u_{j} \notin[0, L]\right] .
\end{aligned}
$$

We can use the self-similarity property and the independence of $\epsilon$ and the $X_{j}$ to write the probabilities under consideration as convolutions which then allows us to deduce

$$
\sum_{j=-\infty}^{\infty} \mathbb{P}\left[X_{j}^{\alpha, c}(t)+a(j-\epsilon) \in[0, L]\right]=\int_{-\infty}^{\infty} \int_{0}^{L / a} p_{t / a^{\alpha}}(x, y) \mathrm{d} y \mathrm{~d} x .
$$

Hence

$$
\begin{aligned}
& V_{t}^{\alpha, c, a}[L] \\
& =\underbrace{\int_{-\infty}^{\infty} \int_{0}^{L / a} p_{t / a^{\alpha}}(x, y) \mathrm{d} y \mathrm{~d} x}_{T_{1}}-\underbrace{\int_{-\infty}^{\infty} \int_{0}^{L / a} \int_{0}^{L / a} p_{t / a^{\alpha}}(x, y) p_{t / a^{\alpha}}(x, z) \mathrm{d} z \mathrm{~d} y \mathrm{~d} x}_{T_{2}} .
\end{aligned}
$$

By Fubini's Theorem and symmetry we have

$$
T_{1}=\int_{0}^{L / a} \int_{-\infty}^{\infty} p_{t / a^{\alpha}}(y, x) \mathrm{d} x \mathrm{~d} y=\frac{L}{a} .
$$

For the other term we make use of the Chapman-Kolmogorov identity and the spatial homogeneity before performing an integral switch to obtain

$$
\begin{aligned}
T_{2} & =\int_{0}^{L / a} \int_{-y}^{(L / a)-y} p_{2 t / a^{\alpha}}(0, z) \mathrm{d} z \mathrm{~d} y \\
& =\frac{L}{a} \int_{-L / a}^{L / a} p_{2 t / a^{\alpha}}(0, z) \mathrm{d} z+\int_{-L / a}^{0} z p_{2 t / a^{\alpha}}(0, z) \mathrm{d} z-\int_{0}^{L / a} z p_{2 t / a^{\alpha}}(0, z) \mathrm{d} z .
\end{aligned}
$$

From the symmetry property we know that, for each $t, p_{t}(0, z)$ is an even function in $z$ and $g(z):=z p_{t}(0, z)$ is an odd function in $z$. These facts allow us to conclude, bringing the two terms together,

$$
V_{t}^{\alpha, c, a}[L]=\frac{2 L}{a} \int_{L / a}^{\infty} p_{2 t / a^{\alpha}}(0, z) \mathrm{d} z+2 \int_{0}^{L / a} z p_{2 t / a^{\alpha}}(0, z) \mathrm{d} z .
$$

Applying Fourier inversion to the characteristic function $\phi_{X^{\alpha, c}(t)}(\theta)$ given at (2.1), we deduce that the transition density can be expressed as

$$
p_{t}(0, z \mid \alpha, c)=\frac{1}{\pi} \int_{0}^{\infty} \cos (z \theta) e^{-c t \theta^{\alpha}} \mathrm{d} \theta .
$$

Using this density, the symmetry property and the expression (2.9) we obtain

$$
V_{t}^{\alpha, c, a}[L]=\frac{L}{a}+\frac{2}{\pi} \int_{0}^{\infty} \frac{e^{-2 c t(\theta / a)^{\alpha}}}{\theta^{2}}\left[\cos \left(\frac{L \theta}{a}\right)-1\right] \mathrm{d} \theta .
$$


Now making the change of variable $u=L \theta / a$, using $\int_{0}^{\infty} \sin ^{2}(u / 2) / u^{2} d u=\pi / 4$ and a double angle formula yields the given alternative expression for the number variance.

Having found a general expression for the number variance we are able to consider its behaviour as the interval length $L$ is increased. Note that given positive real valued functions $g$ and $h$ we let $g \sim h$ signify that $\lim \frac{g}{h}=1$.

Theorem 2.3. Consider the number variance $V_{t}^{\alpha, c, a}[L]$ for the system of symmetric $\alpha$-stable processes considered above.

$$
V_{t}^{\alpha, c, a}[L] \sim \begin{cases}k_{\alpha} \frac{2 c t}{a} L^{1-\alpha}+\kappa_{\text {sat }}(\alpha, c, a, t), & \text { for } \alpha \in(0,1) \cup(1,2), \\ k_{\alpha} \frac{2 c t}{a} \log (L)+\kappa_{\text {sat }}(\alpha, c, a, t), & \text { for } \alpha=1, \\ \frac{a}{\sqrt{2 c \pi t}} e^{-L^{2} / 8 c t}+\kappa_{\text {sat }}(\alpha, c, a, t), & \text { for } \alpha=2,\end{cases}
$$

as $L \rightarrow \infty$, with $k_{\alpha}=\left(\int_{0}^{\infty} x^{-\alpha} \sin x \mathrm{~d} x\right)^{-1}$ and

$$
\kappa_{\text {sat }}(\alpha, c, a, t)=\frac{2}{a \pi}(2 t c)^{1 / \alpha} \Gamma\left(1-\frac{1}{\alpha}\right),
$$

where $\Gamma(x):=\int_{0}^{\infty} s^{x-1} e^{-s} \mathrm{~d} s$ is the usual Gamma function.

Proof. From (2.9) note that we may re-write the expression for the number variance as

$$
V_{t}^{\alpha, c, a}[L]=\frac{L}{a} \mathbb{P}\left[\left|X^{\alpha, c}\left(\frac{2 t}{a^{\alpha}}\right)\right|>L / a\right]+\mathbb{E}_{\mathbb{P}}\left[\left|X^{\alpha, c}\left(\frac{2 t}{a^{\alpha}}\right)\right| ;\left|X^{\alpha, c}\left(\frac{2 t}{a^{\alpha}}\right)\right|<L / a\right],
$$

Now the behaviour of the first term in this expression is well known (see (23), page 16). For $\alpha \in(0,2)$ we have

$$
\frac{L}{a} \mathbb{P}\left[\left|X^{\alpha, c}\left(\frac{2 t}{a^{\alpha}}\right)\right|>L / a\right] \sim k_{\alpha} \frac{2 c t}{a} L^{1-\alpha} \quad \text { as } L \rightarrow \infty,
$$

where $k_{\alpha}$ is as above. When $\alpha=1$, the $L^{1-\alpha}$ in the expression is replaced by $\log (L)$. In the Gaussian case $(\alpha=2)$ we have instead

$$
\frac{L}{a} \mathbb{P}\left[\left|X\left(\frac{2 t}{a^{2}}\right)\right|>L / a\right] \sim \frac{a}{\sqrt{2 c \pi t}} e^{-L^{2} / 8 c t} \quad \text { as } \quad L \rightarrow \infty .
$$

To deal with the second term of (2.12) observe that we have

$$
\begin{aligned}
\mathbb{E}_{\mathbb{P}}\left[\left|X^{\alpha, c}\left(2 t / a^{\alpha}\right)\right| ;\left|X^{\alpha, c}\left(2 t / a^{\alpha}\right)\right|<L / a\right] & \stackrel{L \rightarrow \infty}{\rightarrow} \mathbb{E}_{\mathbb{P}}\left[\left|X^{\alpha, c}\left(2 t / a^{\alpha}\right)\right|\right] \\
& =\int_{0}^{\infty} \mathbb{P}\left[\left|X^{\alpha, c}\left(2 t / a^{\alpha}\right)\right|>\lambda\right] \mathrm{d} \lambda .
\end{aligned}
$$

Thus it is clear that the rate of divergence or saturation of the number variance is determined by the upper tail of the distribution of the underlying symmetric $\alpha$-stable process. Consequently, for $\alpha \in(1,2]$ (the saturating cases)

$$
\lim _{L \rightarrow \infty} V_{t}^{\alpha, c, a}[L]=\mathbb{E}_{\mathbb{P}}\left[\left|X^{\alpha, c}\left(\frac{2 t}{a^{\alpha}}\right)\right|\right]=: \kappa_{\text {sat }}(\alpha, c, a, t)<\infty .
$$


The exact expression for $\kappa_{\text {sat }}$ is obtained from the moments. By (25), if $X$ is a symmetric $\alpha$-stable random variable with $0<\alpha \leq 2$ and scale $\sigma$, then for $-1<\delta<\alpha$ we have

$$
\mathbb{E}\left[|X|^{\delta}\right]=\frac{\sigma^{\delta / \alpha} 2^{\delta} \Gamma\left(\frac{1+\delta}{2}\right) \Gamma(1-\delta / \alpha)}{\Gamma(1 / 2) \Gamma(1-\delta / 2)} .
$$

Applying this theorem with $\delta=1, \sigma=\frac{2 c t}{a^{\alpha}}$ gives (2.11). To see how this fits in with the integral expression for $V_{t}^{\alpha, c, a}[L]$, it may be verified that for $\alpha \in(1,2]$

$$
\kappa_{\text {sat }}(\alpha, c, a, t)=-\frac{2}{\pi} \int_{0}^{\infty} \frac{e^{-2 c t(\theta / a)^{\alpha}}}{\theta^{2}} \mathrm{~d} \theta .
$$

The saturation result is now a consequence of Theorem 2.3 ,

Corollary 2.4. 1. If $\alpha \in(0,1]$, at each time $t>0, V_{t}^{\alpha, c, a}[L]$ diverges as $L \rightarrow \infty$.

2. If $\alpha \in(1,2]$, at each time $t<\infty, V_{t}^{\alpha, c, a}[L]$ saturates to the level $\kappa_{\text {sat }}(\alpha, c, a, t)$ as $L \rightarrow \infty$.

Remark 2.5. Even from the simplest equation (2.6) it is clear that the largest contributions to the number variance come from the activity at the edges of the interval under consideration. Thus intuitively, the fatter the tails of the distributions concerned the greater the number of particles that may be in the vicinity of these edges making these substantial contributions and consequently the slower the decay in the growth of the number variance.

We now apply Theorems 2.2 and 2.3 to two well known examples.

Corollary 2.6 (Brownian case). Consider an infinite system of Brownian particles started from the initial configuration $\left(u_{j}\right)_{j=-\infty}^{\infty}$ as given at (2.2). The number variance for this process is

$$
V_{t}^{2, \frac{1}{2}, a}[L]=\frac{2}{a}\left[L \Phi\left(\frac{-L}{\sqrt{2 t}}\right)+\sqrt{\frac{t}{\pi}}\left(1-e^{-L^{2} / 4 t}\right)\right],
$$

where

$$
\Phi(x):=\frac{1}{\sqrt{2 \pi}} \int_{-\infty}^{x} e^{-y^{2} / 2} \mathrm{~d} y .
$$

As $L \rightarrow \infty$ this number variance saturates exponentially quickly to the level

$$
\frac{2}{a} \sqrt{\frac{t}{\pi}}
$$

Corollary 2.7 (Symmetric Cauchy case). Consider an infinite system of symmetric Cauchy processes started from the initial configuration $\left(u_{j}\right)_{j=-\infty}^{\infty}$ as given at (2.2). The number variance for this process is

$$
V_{t}^{1,1, a}[L]=\frac{L}{a}\left[1-\frac{2}{\pi} \arctan \left[\frac{L}{2 t}\right]\right]+\frac{2 t}{a \pi}\left[\log \left(1+\left(\frac{L}{2 t}\right)^{2}\right)\right] .
$$

For "large" (relative to 2t) L, we have

$$
V_{t}^{1,1, a}[L] \approx \frac{4 t}{a \pi} \log \left(\frac{L}{2 t}\right)
$$

and so the number variance diverges at a logarithmic rate as $L \rightarrow \infty$. 
Remark 2.8. Coincidentally, in the symmetric Cauchy case, if we set $a=1, t=\frac{1}{4 \pi}$ we have

$$
V_{\frac{1}{4 \pi}}^{1,1,1}[L] \approx \frac{1}{\pi^{2}} \log (2 \pi L)
$$

and so we see similar number variance behaviour to that in the sine kernel case (1.2).

Remark 2.9. The Cauchy $(\alpha=1)$ case has the slowest growing non-saturating number variance amongst all those considered here. Analogously, the sine kernel determinantal process has the slowest growing number variance amongst all translation invariant determinantal processes whose kernels correspond to projections (i.e. the Fourier transform of the kernel is an indicator) see (27).

Remark 2.10. At the other extreme note that as $\alpha \rightarrow 0$ we recover Poisson behaviour in that

$$
\lim _{\alpha \rightarrow 0} V_{t}^{\alpha, c, a}[L]=\frac{L}{a}\left(1-e^{-2 c t}\right) .
$$

\subsection{A Poisson approximation for the counting function}

At the beginning of this section we recalled that a system of independent processes (satisfying fairly general conditions) started from a Poisson process on $\mathbb{R}$ remains in a Poisson process configuration and hence demonstrates a number variance linear in L, for all time. Now from (2.7) and (2.8) we deduce that

$$
V_{t}^{\alpha, c, a}[L] \leq L / a, \quad \forall \alpha, c, a, t .
$$

From the integral expression for the number variance (2.4), we see that for fixed $L$

$$
V_{t}^{\alpha, c, a}[L] \rightarrow L / a \quad \text { as } t \rightarrow \infty,
$$

and for each $t$, as $L$ is decreased

$$
V_{t}^{\alpha, c, a}[L] \stackrel{L \rightarrow 0}{\sim} L / a .
$$

So in both these limiting cases (as well as in the $\alpha \rightarrow 0$ case c.f. Remark 2.10) the maximal linear number variance is attained. The balance of the parameters $L, a, \alpha$ and $t$, encapsulated by the number variance, determines how "far away" from being Poisson the distribution of $N_{t}^{\alpha, c, a}[0, L]$ actually is. Below we make this observation precise.

Recall that given a measurable space $(\Omega, \mathcal{F})$ we define the total variation distance $d_{T V}(\cdot, \cdot)$ between two probability measures $\mu_{1}, \mu_{2}$ on $\Omega$ by

$$
d_{T V}\left(\mu_{1}, \mu_{2}\right):=\sup _{F \in \mathcal{F}}\left|\mu_{1}(F)-\mu_{2}(F)\right| .
$$

Propostition 2.11. Let $\mathcal{L}\left(N_{t}^{\alpha, c, a}[0, L]\right)$ denote the law of the random variable $N_{t}^{\alpha, c, a}[0, L]$ defined at (2.5). Let $P o(L / a)$ denote the law of a Poisson random variable with mean $L / a$. Then, for $L \geq 1$,

$$
\frac{L-V_{t}^{\alpha, c, a}[L] a}{32 L} \leq d_{T V}\left(\mathcal{L}\left(N_{t}^{\alpha, c, a}[0, L]\right), P o(L / a)\right) \leq\left(1-e^{-\frac{L}{a}}\right) \frac{L-V_{t}^{\alpha, c, a}[L] a}{L} .
$$


Proof. The result is an application of a theorem of Barbour and Hall (2). Their theorem states that if $A:=\sum_{j} \mathbb{I}_{j}$ is the sum of independent indicator random variables indexed by $j$ and $q_{j}(L)=\mathbb{E}\left[\mathbb{I}_{j}\right], \lambda=\sum_{j} q_{j}(L)$ then if we denote the law of A by $\mathcal{L}(A)$ we have

$$
\frac{1}{32} \min (1,1 / \lambda) \sum_{j} q_{j}(L)^{2} \leq d_{T V}(\mathcal{L}(A), \operatorname{Po}(L / a)) \leq \frac{1-e^{-\lambda}}{\lambda} \sum_{j} q_{j}(L)^{2} .
$$

In our specific case we have $N_{t}^{\alpha, c, a}[0, L]$ as the sum of independent indicator random variables given by (2.5), $\lambda=L / a$ and $\sum_{j} q_{j}(L)^{2}=\frac{L}{a}-V_{t}^{\alpha, c, a}[L]$.

Remark 2.12. For a fixed $t$, the Poisson approximation becomes less accurate as $L \rightarrow \infty$. The greater the value of $\alpha$ the faster the quality of the approximation deteriorates. For $\alpha>1$, due to the fact that the number variance saturates, the approximation of the law of $N_{t}^{\alpha, c, a}[0, L]$ by a Poisson distribution of mean $L / a$ becomes very poor with the total variation distance clearly bounded away from zero.

\section{Gaussian fluctuations of the counting function}

Thus far we have been concerned with the variance of the counting function $N_{t}^{\alpha, c, a}[0, L]$ (2.5). Of course this variance is, by definition, a description of the fluctuation of $N_{t}^{\alpha, c, a}[0, L]$ around its mean $\frac{L}{a}$. In this section we will further characterize these fluctuations.

Propostition 3.1. Let $N_{t}^{\alpha, c, a}[0, L], V_{t}^{\alpha, c, a}[L]$ denote, as usual, the counting function and number variance. For the cases with $\alpha \in(0,1]$ we have that

$$
\frac{N_{t}^{\alpha, c, a}[0, L]-L / a}{\sqrt{V_{t}^{\alpha, c, a}[L]}}
$$

converges in distribution to a standard normal random variable as $L \rightarrow \infty$.

Proof. Recall that the cumulants $c_{k}, k \in \mathbb{N}$ of a real valued random variable $Y$ are defined by

$$
\log \mathbb{E}[\exp (i \theta Y)]=\sum_{k=1}^{\infty} c_{k} \frac{(i \theta)^{k}}{k !} .
$$

Using the independence of the individual symmetric $\alpha$-stable processes and then applying the Taylor expansion of $\log (1+x)$ about zero, we have

$$
\begin{aligned}
\log \mathbb{E}_{\mathbb{P}}\left[\exp \left(i \theta N_{t}^{\alpha, c, a}[0, L]\right)\right] & =\sum_{j=-\infty}^{\infty} \log \left[\left(e^{i \theta}-1\right) q_{j}(L)+1\right] \\
& =\sum_{m=1}^{\infty} \frac{\left(e^{i \theta}-1\right)^{m}}{m}(-1)^{m+1}\left(\sum_{j=-\infty}^{\infty} q_{j}(L)^{m}\right),
\end{aligned}
$$

where $q_{j}(L):=\mathbb{P}\left[X_{j}^{\alpha, c}(t)+u_{j} \in[0, L]\right]$ and $X_{j}^{\alpha, c}(t)$ denotes as usual the underlying symmetric $\alpha$-stable process labelled by $j$ with $u_{j}$ the corresponding starting position. Hence, the cumulants of $N_{t}^{\alpha, c, a}[0, L]$ are given by

$$
c_{k}=\left.\frac{\mathrm{d}^{k}}{\mathrm{~d} \theta^{k}} \sum_{m=1}^{\infty} \frac{\left(e^{i \theta}-1\right)^{m}}{m}(-1)^{m+1}\left(\sum_{j=-\infty}^{\infty} q_{j}(L)^{m}\right)\right|_{\theta=0} .
$$


It is straightforward to see that

$$
c_{1}=L / a, \quad c_{2}=\sum_{j=-\infty}^{\infty} q_{j}(L)-q_{j}(L)^{2}=V_{t}^{\alpha, c, a}[L]
$$

give the mean and number variance respectively. More generally from the equation (3.2) it is possible to deduce the following recursive relation

$$
c_{k}=\sum_{m=2}^{k-1} \beta_{k, m} c_{m}+(-1)^{k}(k-1) ! \sum_{j=-\infty}^{\infty} q_{j}(L)-q_{j}(L)^{k},
$$

where $\beta_{k, m}$ are constant, finite, combinatorial coefficients which will not be needed here. Now let

$$
Y_{t}^{\alpha, c, a}:=\frac{N_{t}^{\alpha, c, a}[0, L]-L / a}{\sqrt{V_{t}^{\alpha, c, a}[L]}} .
$$

It is easily deduced that the cumulants $\tilde{c_{k}}, k \in \mathbb{N}$ of $Y_{t}^{\alpha, c, a}$ are given by

$$
\begin{aligned}
& \tilde{c_{1}}=0, \\
& \tilde{c}_{k}=\frac{c_{k}}{\left(V_{t}^{\alpha, c, a}[L]\right)^{k / 2}}, \quad \text { for } k \geq 2 .
\end{aligned}
$$

To prove the Proposition it is sufficient to show that in the limit as $L \rightarrow \infty$, the cumulants correspond to those of a Gaussian random variable. That is, we have $\tilde{c}_{3}=\tilde{c}_{4}=\tilde{c}_{5}=\cdots=0$. Equivalently, we need to show

$$
c_{k}=o\left(\left(V_{t}^{\alpha, c, a}[L]\right)^{k / 2}\right)=o\left(c_{2}^{k / 2}\right) \quad \text { as } L \rightarrow \infty, \text { for } k \geq 3 .
$$

We use an induction argument.

Suppose that $c_{m}=o\left(c_{2}^{m / 2}\right)$ for $m=3, \ldots, k-1$. Assume, without loss of generality, that $k$ is even. We use the inequality

$$
\begin{aligned}
q_{j}(L)-q_{j}(L)^{k} & =\sum_{l=1}^{k-1} q_{j}(L)^{l}-q_{j}(L)^{l+1} \\
& \leq(k-1)\left(q_{j}(L)-q_{j}(L)^{2}\right),
\end{aligned}
$$

in conjunction with the recursive relation for $c_{k}$ given by (3.3) to deduce

$$
\sum_{m=2}^{k-1} \beta_{k, m} c_{m} \leq c_{k} \leq(k-1) !(k-1) c_{2}+\sum_{m=2}^{k-1} \beta_{k, m} c_{m} .
$$

From our induction supposition this implies that

$$
o\left(c_{2}^{k / 2}\right) \leq c_{k} \leq o\left(c_{2}^{k / 2}\right)+(k-1) !(k-1) c_{2} .
$$

However, from the results of the previous section, we know that, for these cases with $\alpha \in(0,1]$, for $k \geq 3, c_{2}^{\frac{k-2}{2}}=V_{t}^{\alpha, c, a}[L]^{\frac{k-2}{2}} \rightarrow \infty$ as $L \rightarrow \infty$. Thus from (3.5) $c_{k}=o\left(c_{2}^{k / 2}\right)$ also. Now using the same arguments as for the inequality (3.4) we find

$$
-\frac{1}{\sqrt{c_{2}}} \leq \frac{c_{3}}{c_{2}^{3 / 2}} \leq \frac{1}{\sqrt{c_{2}}}
$$


Thus we have $c_{3}=o\left(c_{2}^{3 / 2}\right)$. By the induction argument we can deduce that $\frac{c_{k}}{\left(c_{2}\right)^{k / 2}} \rightarrow 0$ as $L \rightarrow \infty$ for all $k \geq 3$ which concludes the proof.

Remark 3.2. The divergence of the number variance is relied upon in a similar way to prove the analogous Gaussian fluctuation results for a large class of determinantal processes, see (8; 28; 27). Here we have adapted the "Costin-Lebowitz-Soshnikov" method to this non-determinantal setting. We note that the Proposition could also have been proved by applying the LindbergFeller Central Limit Theorem (see e.g. (10)).

Proposition 3.1 applies to the cases with $\alpha \in(0,1]$. The following convergence in distribution result applies to all cases with $\alpha \in(0,2]$ and is obtained by allowing both interval length and time $t$ tend to infinity together in an appropriate way.

Propostition 3.3. For any fixed $s \in[0, \infty)$ we have that

$$
\frac{N_{t}^{\alpha, c, a}\left[0, s t^{1 / \alpha}\right]-s t^{1 / \alpha} / a}{t^{1 / 2 \alpha}}
$$

converges in distribution as $t \rightarrow \infty$, to a normal random variable with zero mean and variance $f^{\alpha, c, a}(s)$, where

$$
\begin{aligned}
f^{\alpha, c, a}(s) & :=V_{1}^{\alpha, c, a}[s] \\
& =\frac{4 s}{a \pi} \int_{0}^{\infty} \frac{\sin ^{2}(u / 2)}{u^{2}}\left(1-e^{-2 c(u / s)^{\alpha}}\right) \mathrm{d} u
\end{aligned}
$$

Proof. Since $V_{t}^{\alpha, c, a}\left[s t^{1 / \alpha}\right] \rightarrow \infty$ as $t \rightarrow \infty$, a similar argument as for the proof of Proposition 3.1 allows us to conclude that

$$
\frac{N_{t}^{\alpha, c, a}\left[0, s t^{1 / \alpha}\right]-s t^{1 / \alpha} / a}{\sqrt{V_{t}^{\alpha, c, a}\left[s t^{1 / \alpha}\right]}}
$$

converges in distribution as $t \rightarrow \infty$ to a standard normal random variable. From the integral expression for the number variance (2.4) we have

$$
\frac{V_{t}^{\alpha, c, a}\left[s t^{1 / \alpha}\right]}{t^{1 / \alpha}}=\frac{4 s}{a \pi} \int_{0}^{\infty} \frac{\sin ^{2}(u / 2)}{u^{2}}\left(1-e^{-2 c(u / s)^{\alpha}}\right) \mathrm{d} u=: f^{\alpha, c, a}(s) .
$$

Note that from (2.14) we know $f^{\alpha, c, a}(s)<\infty$ for all $s<\infty$ and so the result follows from the scaling property of the Gaussian distribution.

\section{The fluctuation process}

We proceed by adding some dynamics to the fluctuations of the counting function and define, for each $\alpha \in(0,2], c>0, a \in \mathbb{R}_{+}$, the process

$$
Z_{t}^{\alpha, c, a}(s):=\frac{N_{t}^{\alpha, c, a}\left[0, s t^{1 / \alpha}\right]-s t^{1 / \alpha} / a}{t^{1 / 2 \alpha}}, s \in[0, \infty) .
$$

Our aim is to consider the limit process obtained as $t \rightarrow \infty$. 


\subsection{The covariance structure}

We begin to characterize these processes by identifying their covariance structure.

Lemma 4.1. $\left\{Z_{t}^{\alpha, c, a}(s) ; s \in[0, \infty)\right\}$ has covariance structure

$$
\operatorname{Cov}\left(Z_{t}^{\alpha, c, a}(r), Z_{t}^{\alpha, c, a}(s)\right)=\frac{1}{2}\left(f^{\alpha, c, a}(s)+f^{\alpha, c, a}(r)-f^{\alpha, c, a}(|r-s|)\right) .
$$

Proof. By construction

$$
N_{t}^{\alpha, c, a}\left[0,(r \vee s) t^{1 / \alpha}\right]-N_{t}^{\alpha, c, a}\left[0,(r \wedge s) t^{1 / \alpha}\right] \stackrel{\text { dist }}{=} N_{t}^{\alpha, c, a}\left[0,|r-s| t^{1 / \alpha}\right] .
$$

Hence, from the definition of $Z_{t}^{\alpha, c, a}$

$$
Z_{t}^{\alpha, c, a}(|r-s|) \stackrel{\text { dist }}{=} Z_{t}^{\alpha, c, a}(r \vee s)-Z_{t}^{\alpha, c, a}(r \wedge s),
$$

which implies that

$$
\begin{array}{r}
\operatorname{Var}\left(Z_{t}^{\alpha, c, a}(|r-s|)\right)=\operatorname{Var}\left(Z_{t}^{\alpha, c, a}(r \wedge s)\right)+\operatorname{Var}\left(Z_{t}^{\alpha, c, a}(r \vee s)\right) \\
-2 \operatorname{Cov}\left(Z_{t}^{\alpha, c, a}(r \wedge s), Z_{t}^{\alpha, c, a}(r \vee s)\right) .
\end{array}
$$

Rearranging gives

$$
\begin{aligned}
& \operatorname{Cov}\left(Z_{t}^{\alpha, c, a}(s), Z_{t}^{\alpha, c, a}(r)\right) \\
& \quad=\frac{1}{2}\left(\operatorname{Var}\left(Z_{t}^{\alpha, c, a}(r \wedge s)\right)+\operatorname{Var}\left(Z_{t}^{\alpha, c, a}(r \vee s)\right)-\operatorname{Var}\left(Z_{t}^{\alpha, c, a}(|r-s|)\right)\right) \\
& =\frac{1}{2 t^{1 / \alpha}}\left(V_{t}^{\alpha, c, a}\left[s t^{1 / \alpha}\right]+V_{t}^{\alpha, c, a}\left[r t^{1 / \alpha}\right]-V_{t}^{\alpha, c, a}\left[|r-s| t^{1 / \alpha}\right]\right)
\end{aligned}
$$

On referring back to the definition of $f^{\alpha, c, a}(\cdot)$ we see that this last statement is equivalent to the result of the Lemma.

Note that the covariance does not depend on t.

\subsection{Convergence of finite dimensional distributions}

Given the covariance structure of $Z_{t}^{\alpha, c, a}$ and the identification of its Gaussian one dimensional marginal distributions the natural next step is to consider the finite dimensional distributions.

Propostition 4.2. Let $\left\{G^{\alpha, c, a}(s): s \in[0, \infty)\right\}$ be a centered Gaussian process with covariance structure

$$
\operatorname{Cov}\left(G^{\alpha, c, a}\left(s_{i}\right), G^{\alpha, c, a}\left(s_{j}\right)\right)=\frac{1}{2}\left(f^{\alpha, c, a}\left(s_{i}\right)+f^{\alpha, c, a}\left(s_{j}\right)-f^{\alpha, c, a}\left(\left|s_{i}-s_{j}\right|\right)\right) .
$$

For any $0 \leq s_{1} \leq s_{2} \leq \cdots \leq s_{n}<\infty$ we have

$$
\left(Z_{t}^{\alpha, c, a}\left(s_{1}\right), Z_{t}^{\alpha, c, a}\left(s_{2}\right), \ldots, Z_{t}^{\alpha, c, a}\left(s_{n}\right)\right) \Rightarrow\left(G^{\alpha, c, a}\left(s_{1}\right), G^{\alpha, c, a}\left(s_{2}\right), \ldots, G^{\alpha, c, a}\left(s_{n}\right)\right)
$$

as $t \rightarrow \infty$. 
Proof. As previously noted, the mean and covariance structure of $Z_{t}^{\alpha, c, a}(s)$ are not dependent on t. Therefore, all that remains is to show that, in the limit as $t \rightarrow \infty$, the joint distributions are Gaussian. We again make use of the cumulants.

Recall that given a random vector $\mathbf{Y}:=\left(Y_{1}, Y_{2}, \ldots, Y_{n}\right) \in \mathbb{R}^{n}$, the joint cumulants of $\mathbf{Y}$ denoted $C_{m_{1}, m_{2}, \ldots, m_{n}}(\mathbf{Y})$ are defined via the $m_{j}{ }^{\prime}$ th partial derivatives of the logarithm of the characteristic function of $\mathbf{Y}$. That is,

$$
C_{m_{1}, m_{2}, \ldots, m_{n}}(\mathbf{Y}):=\left.\left(\frac{\partial}{\partial\left(i \theta_{1}\right)}\right)^{m_{1}}\left(\frac{\partial}{\partial\left(i \theta_{2}\right)}\right)^{m_{2}} \cdots\left(\frac{\partial}{\partial\left(i \theta_{n}\right)}\right)^{m_{n}} \log \mathbb{E}\left[\exp \left(\sum_{j=1}^{n} i \theta_{j} Y_{j}\right)\right]\right|_{\theta=\mathbf{0}} .
$$

If

$$
\begin{aligned}
& C_{0,0, \ldots, \underbrace{1}_{\mathrm{i} \text { 'th }}, \ldots, 0}(\mathbf{Y})=\mathbb{E}\left[Y_{i}\right]=0 \\
& C_{0,0, \ldots, \underbrace{2}_{\mathbf{i}^{\prime} \text { th }}, \ldots, 0}(\mathbf{Y})=\operatorname{Var}\left[Y_{i}\right]=\Sigma_{i i}
\end{aligned}
$$

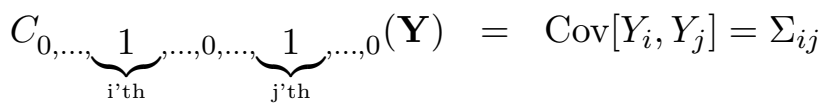

and in particular

$$
C_{m_{1}, m_{2}, \ldots, m_{n}}(\mathbf{Y})=0, \quad \text { whenever } \quad \sum_{i=1}^{n} m_{i} \geq 3
$$

then $\mathbf{Y}$ has a multivariate normal $(0, \Sigma)$ distribution. To prove the Proposition it is enough to show that

$$
\left(Z_{t}^{\alpha, c, a}\left(s_{1}\right), Z_{t}^{\alpha, c, a}\left(s_{2}\right)\right) \rightarrow \text { MultivariateNormal }\left(0, \Sigma^{\alpha, c, a}\right)
$$

in distribution as $t \rightarrow \infty$, where $\Sigma^{\alpha, c, a}$ is the $2 \times 2$ covariance matrix

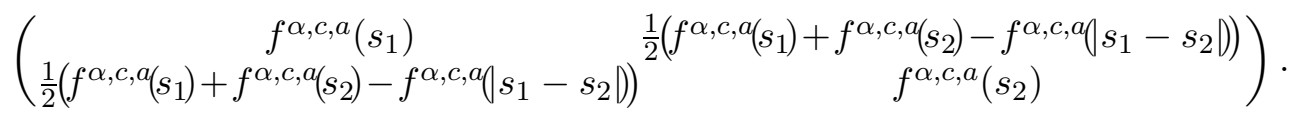

We begin by computing the characteristic function of

$$
\left(N_{t}^{\alpha, c, a}\left[0, s_{1} t^{1 / \alpha}\right], N_{t}^{\alpha, c, a}\left[0, s_{2} t^{1 / \alpha}\right]\right) .
$$

Using the independence of the individual particles we have

$$
\begin{aligned}
& \mathbb{E}_{\mathbb{P}}\left[\exp \left(i \theta_{1} N_{t}^{\alpha, c, a}\left[0, s_{1} t^{1 / \alpha}\right]+i \theta_{2} N_{t}^{\alpha, c, a}\left[0, s_{2} t^{1 / \alpha}\right]\right)\right] \\
& =\mathbb{E}_{\mathbb{P}}\left[\exp i\left(\left(\theta_{1}+\theta_{2}\right) N_{t}^{\alpha, c, a}\left[0, s_{1} t^{1 / \alpha}\right]+\theta_{2} N_{t}^{\alpha, c, a}\left[s_{1} t^{1 / \alpha}, s_{2} t^{1 / \alpha}\right]\right)\right] \\
& =\prod_{j=-\infty}^{\infty}\left(e^{i\left(\theta_{1}+\theta_{2}\right)} \mathbb{P}\left[X_{j}^{\alpha, c}(t)+u_{j} \in\left[0, s_{1} t^{1 / \alpha}\right]\right]\right. \\
& \left.\quad+e^{i \theta_{2}} \mathbb{P}\left[X_{j}^{\alpha, c}(t)+u_{j} \in\left[s_{1} t^{1 / \alpha}, s_{2} t^{1 / \alpha}\right]\right]+\mathbb{P}\left[X_{j}^{\alpha, c}(t)+u_{j} \notin\left[0, s_{2} t^{1 / \alpha}\right]\right]\right) .
\end{aligned}
$$


For ease of notation we will henceforth let

$$
q_{j}\left(s_{l}, s_{r}\right):=\mathbb{P}\left[X_{j}^{\alpha, c}(t)+u_{j} \in\left[s_{l} t^{1 / \alpha}, s_{r} t^{1 / \alpha}\right]\right], \quad 0 \leq s_{l} \leq s_{r}<\infty .
$$

The joint cumulants are given by

$$
\begin{aligned}
& C_{m_{1}, m_{2}}\left(N_{t}^{\alpha, c, a}\left[0, s_{1} t^{1 / \alpha}\right], N_{t}^{\alpha, c, a}\left[0, s_{2} t^{1 / \alpha}\right]\right) \\
& =\left.\sum_{j=\infty}^{\infty}\left(\frac{\partial}{\partial\left(i \theta_{1}\right)}\right)^{m_{1}}\left(\frac{\partial}{\partial\left(i \theta_{2}\right)}\right)^{m_{2}} \log \left(e^{i\left(\theta_{1}+\theta_{2}\right)} q_{j}\left(0, s_{1}\right)+e^{i \theta_{2}} q_{j}\left(s_{1}, s_{2}\right)+1-q_{j}\left(0, s_{2}\right)\right)\right|_{\theta=0} .
\end{aligned}
$$

Now using the fact that

$$
\begin{aligned}
& \left(\frac{\partial}{\partial\left(i \theta_{1}\right)}\right)^{m_{1}}\left(\frac{\partial}{\partial\left(i \theta_{2}\right)}\right)^{m_{2}} e^{i\left(\theta_{1}+\theta_{2}\right)} q_{j}\left(0, s_{1}\right)+e^{i \theta_{2}} q_{j}\left(s_{1}, s_{2}\right)+1-q_{j}\left(0, s_{2}\right) \\
= & e^{i\left(\theta_{1}+\theta_{2}\right)} q_{j}\left(0, s_{1}\right) \quad \forall m_{1}, m_{2} \text { s.t. } m_{1} \geq 1
\end{aligned}
$$

and

$$
\begin{aligned}
& \left(\frac{\partial}{\partial\left(i \theta_{2}\right)}\right)^{m_{2}} e^{i\left(\theta_{1}+\theta_{2}\right)} q_{j}\left(0, s_{1}\right)+e^{i \theta_{2}} q_{j}\left(s_{1}, s_{2}\right)+1-q_{j}\left(0, s_{2}\right) \\
= & e^{i\left(\theta_{1}+\theta_{2}\right)} q_{j}\left(0, s_{1}\right)+e^{i \theta_{2}} q_{j}\left(s_{1}, s_{2}\right) \quad \forall m_{2},
\end{aligned}
$$

along with

$$
\left.\left(e^{i\left(\theta_{1}+\theta_{2}\right)} q_{j}\left(0, s_{1}\right)+e^{i \theta_{2}} q_{j}\left(s_{1}, s_{2}\right)+1-q_{j}\left(0, s_{2}\right)\right)\right|_{\theta=0}=1,
$$

we deduce the following generalization of the recursive relation (3.3), with obvious short-hand notation for the joint cumulants

$$
\begin{aligned}
C_{m_{1}, m_{2}}= & \sum_{\substack{k, l: \\
2 \leq k+l \leq m_{1}+m_{2}-1}} \beta_{k, l, m_{1}, m_{2}} C_{k, l} \\
& +(-1)^{m_{1}+m_{2}}\left(m_{1}+m_{2}-1\right) ! \sum_{j=-\infty}^{\infty} q_{j}\left(0, s_{1}\right)-q_{j}\left(0, s_{1}\right)^{m_{1}} q_{j}\left(0, s_{2}\right)^{m_{2}} .
\end{aligned}
$$

Now suppose that $C_{k, l}=o\left(t^{\frac{k+l}{2 \alpha}}\right)$ for all $k, l$ such that $k+l \in\left\{3,4, \ldots, m_{1}+m_{2}-1\right\}$ and without loss of generality assume that $m_{1}+m_{2}$ is even. Since

$$
0 \leq q_{j}\left(0, s_{1}\right)-q_{j}\left(0, s_{1}\right)^{m_{1}} q_{j}\left(0, s_{2}\right)^{m_{2}} \leq q_{j}\left(0, s_{1}\right),
$$

if we assume that the above induction hypothesis holds, then we have

$$
o\left(t^{\frac{m_{1}+m_{2}-1}{2 \alpha}}\right) \leq C_{m_{1}, m_{2}} \leq o\left(t^{\frac{m_{1}+m_{2}-1}{2 \alpha}}\right)+\left(m_{1}+m_{2}-1\right) ! \frac{s_{1} t^{1 / \alpha}}{a},
$$

which implies that $C_{m_{1}, m_{2}}=o\left(t^{\frac{m_{1}+m_{2}}{2 \alpha}}\right)$ also. We check the third order joint cumulants directly and deduce that

$$
o\left(t^{\frac{3}{2 \alpha}}\right) \leq C_{k, l} \leq o\left(t^{\frac{3}{2 \alpha}}\right)+2\left(\frac{s_{2} t^{1 / \alpha}}{a}\right) \quad \text { when } k+l=3
$$


since the variances and covariance of $\left(N_{t}^{\alpha, c, a}\left[0, s_{1} t^{1 / \alpha}\right], N_{t}^{\alpha, c, a}\left[0, s_{2} t^{1 / \alpha}\right]\right)$ (i.e. $\left.C_{2,0}, C_{0,2}, C_{1,1}\right)$ grow at most like $t^{1 / \alpha}$ as $t \rightarrow \infty$. Therefore, by induction, whenever $m_{1}+m_{2} \geq 3$ we have

$$
\frac{C_{m_{1}, m_{2}}\left(N_{t}^{\alpha, c, a}\left[0, s_{1} t^{1 / \alpha}\right], N_{t}^{\alpha, c, a}\left[0, s_{2} t^{1 / \alpha}\right]\right)}{t^{\left(m_{1}+m_{2}\right) / 2 \alpha}} \rightarrow 0 \quad \text { as } t \rightarrow \infty .
$$

In terms of the joint cumulants of $Z_{t}^{\alpha, c, a}$ this implies

$$
C_{m_{1}, m_{2}}\left(Z_{t}^{\alpha, c, a}\left(s_{1}\right), Z_{t}^{\alpha, c, a}\left(s_{2}\right)\right) \rightarrow 0 \quad \text { as } t \rightarrow \infty \quad \text { whenever } m_{1}+m_{2} \geq 3
$$

from which the claim (4.2) and the Proposition follow.

\subsection{A functional limit for the fluctuation process}

In order to give a functional limit result we consider a continuous approximation $\left\{\widehat{Z}_{t}^{\alpha, c, a}(s): s \in\right.$ $[0, \infty)\}$ to the process $\left\{Z_{t}^{\alpha, c, a}(s): s \in[0, \infty)\right\}$. Let

$$
\widehat{Z}_{t}^{\alpha, c, a}(s):=\frac{\widehat{N}_{t}^{\alpha, c, a}\left[0, s t^{1 / \alpha}\right]-s t^{1 / \alpha} / a}{t^{1 / 2 \alpha}}
$$

where $\widehat{N}_{t}^{\alpha, c, a}\left[0, s t^{1 / \alpha}\right]$ is defined to be equal to $N_{t}^{\alpha, c, a}\left[0, s t^{1 / \alpha}\right]$ except at the points of discontinuity where we linearly interpolate. Let $C[0,1]$ be the space of continuous real valued functions on $[0,1]$ equipped with the uniform topology. We shall denote the measure induced by $\left\{\widehat{Z}_{t}^{\alpha, c, a}(s)\right.$ : $s \in[0,1]\}$ on the space $(C[0,1], \mathcal{B}(C[0,1]))$ by $Q_{t}^{\alpha, c, a}$. To simplify notation we restrict attention to the interval $[0,1]$ but note that the ensuing functional limit theorem extends trivially to any finite real indexing set. The remainder of this section is devoted to establishing the following weak convergence result.

Theorem 4.3. Let $Q^{\alpha, c, a}$ be the law of the centered Gaussian process $\left\{G^{\alpha, c, a}(s): s \in[0,1]\right\}$ introduced in the statement of Proposition 4.2. Then

$$
Q_{t}^{\alpha, c, a} \Rightarrow Q^{\alpha, c, a}
$$

as $t \rightarrow \infty$.

Proof. Note that by definition

$$
\left|\widehat{Z}_{t}^{\alpha, c, a}(s)-Z_{t}^{\alpha, c, a}(s)\right| \leq \frac{1}{t^{1 / 2 \alpha}} .
$$

Thus, as $t \rightarrow \infty$, the finite dimensional distributions of $\widehat{Z}_{t}^{\alpha, c, a}(s)$ must converge to the finite dimensional distributions of the limiting process $G^{\alpha, c, a}(s)$ to which those of $Z_{t}^{\alpha, c, a}$ converge. Hence, immediately from Proposition 4.2 we have that for any $0 \leq s_{1} \leq s_{2} \leq \cdots \leq s_{n}<\infty$,

$$
\left(\widehat{Z}_{t}^{\alpha, c, a}\left(s_{1}\right), \widehat{Z}_{t}^{\alpha, c, a}\left(s_{2}\right), \ldots, \widehat{Z}_{t}^{\alpha, c, a}\left(s_{n}\right)\right) \Rightarrow\left(G^{\alpha, c, a}\left(s_{1}\right), G^{\alpha, c, a}\left(s_{2}\right), \ldots, G^{\alpha, c, a}\left(s_{n}\right)\right)
$$

as $t \rightarrow \infty$. Therefore, by a well known result of Prohorov's (see e.g. (하) ), the proposed functional limit theorem holds if the sequence of measures $\left\{Q_{t}^{\alpha, c, a}\right\}$ is tight. Indeed this tightness requirement follows from Proposition 4.4 given below. 
The sufficient conditions for tightness verified below are stated in terms of the distributions

$$
\mathbb{P}\left(\widehat{Z}_{t}^{\alpha, c, a} \in A\right)=Q_{t}^{\alpha, c, a}(A) \quad A \in \mathcal{B}(C[0,1]),
$$

and the modulus of continuity, which in this case is defined by

$$
w\left(\widehat{Z}_{t}^{\alpha, c, a}, \delta\right):=\sup _{|s-r| \leq \delta}\left|\widehat{Z}_{t}^{\alpha, c, a}(s)-\widehat{Z}_{t}^{\alpha, c, a}(r)\right|, \quad \delta \in(0,1] .
$$

Propostition 4.4. Given $\epsilon, \lambda>0 \exists \delta>0, t_{0} \in \mathbb{N}$ such that

$$
\mathbb{P}\left[w\left(\widehat{Z}_{t}^{\alpha, c, a}, \delta\right) \geq \lambda\right] \leq \epsilon, \quad \text { for } t \geq t_{0} .
$$

Proposition 4.4 is proven via the following series of Lemmas.

Lemma 4.5. Suppose $0 \leq u \leq r \leq s \leq v \leq 1$, then

$$
\left|\widehat{Z}_{t}^{\alpha, c, a}(s)-\widehat{Z}_{t}^{\alpha, c, a}(r)\right| \leq\left|\widehat{Z}_{t}^{\alpha, c, a}(v)-\widehat{Z}_{t}^{\alpha, c, a}(u)\right|+(v-u) t^{1 / 2 \alpha} .
$$

Proof. Clearly, by construction

$$
0 \leq \widehat{N}_{t}^{\alpha, c, a}\left[0, s t^{1 / \alpha}\right]-\widehat{N}_{t}^{\alpha, c, a}\left[0, r t^{1 / \alpha}\right] \leq \widehat{N}_{t}^{\alpha, c, a}\left[0, v t^{1 / \alpha}\right]-\widehat{N}_{t}^{\alpha, c, a}\left[0, u t^{1 / \alpha}\right] .
$$

Therefore, using the definition of $\widehat{Z}_{t}^{\alpha, c, a}$, we have

$$
0 \leq \widehat{Z}_{t}^{\alpha, c, a}(s)-\widehat{Z}_{t}^{\alpha, c, a}(r)+\frac{(s-r)}{a} t^{1 / 2 \alpha} \leq \widehat{Z}_{t}^{\alpha, c, a}(v)-\widehat{Z}_{t}^{\alpha, c, a}(u)+\frac{(v-u)}{a} t^{1 / 2 \alpha} .
$$

The result follows by rearranging, using the facts $a \in \mathbb{R}_{+},(v-u) \geq(s-r) \geq 0$ and then considering separately each case

$$
\widehat{Z}_{t}^{\alpha, c, a}(s)-\widehat{Z}_{t}^{\alpha, c, a}(r) \geq 0 \quad \text { or } \quad \widehat{Z}_{t}^{\alpha, c, a}(s)-\widehat{Z}_{t}^{\alpha, c, a}(r)<0 .
$$

\section{Lemma 4.6.}

$$
\left|\widehat{Z}_{t}^{\alpha, c, a}(s)-\widehat{Z}_{t}^{\alpha, c, a}(r)\right| \leq \frac{2}{t^{1 / 2 \alpha}}+\left|Z_{t}^{\alpha, c, a}(s)-Z_{t}^{\alpha, c, a}(r)\right| .
$$

Proof. Follows from (4.5) and an application of the triangle inequality.

To obtain results on the distribution of the modulus of continuity for our sequence of processes $\left\{\widehat{Z}_{t}^{\alpha, c, a}\right\}$ we divide the interval $[0,1]$ into $m$ disjoint subintervals of length approximately $\delta$ as follows. Let

$$
0=r_{0}<r_{k_{1}}<\cdots<r_{k_{m-1}}<r_{k_{m}}=1
$$

where we define

$$
\begin{aligned}
r_{i} & :=\frac{i}{t^{1 / \alpha}}, \quad i \in \mathbb{N} \\
k_{j} & :=j\left\lceil\delta t^{1 / \alpha}\right\rceil, \quad j \in\{0,1,2, \ldots, m-1\}
\end{aligned}
$$

and $\lceil\cdot\rceil$ denotes the ceiling function. We have

$$
\delta \leq r_{k_{j}}-r_{k_{j-1}} \leq \delta+\frac{1}{t^{1 / \alpha}} \quad j \in\{1,2, \ldots, m-1\},
$$

with the subintervals $\left[r_{i-1}, r_{i}\right], i \in \mathbb{N}$ typically being shorter in length. 


\section{Lemma 4.7.}

$$
\mathbb{P}\left[w\left(\widehat{Z}_{t}^{\alpha, c, a}, \delta\right) \geq \lambda\right] \leq \sum_{j=0}^{m-1} \mathbb{P}\left[\max _{k_{j} \leq i \leq k_{j+1}}\left|Z_{t}^{\alpha, c, a}\left(r_{i}\right)-Z_{t}^{\alpha, c, a}\left(r_{k_{j}}\right)\right| \geq \frac{\lambda}{9}-\frac{7}{3 t^{1 / 2 \alpha}}\right] .
$$

Proof. Given the partition (4.7), standard methods (see Theorem 7.4 of (므)) yield

$$
\mathbb{P}\left[w\left(\widehat{Z}_{t}^{\alpha, c, a}, \delta\right) \geq \lambda\right] \leq \sum_{j=0}^{m-1} \mathbb{P}\left[\sup _{r_{k_{j}} \leq s \leq r_{k_{j+1}}}\left|\widehat{Z}_{t}^{\alpha, c, a}(s)-\widehat{Z}_{t}^{\alpha, c, a}\left(r_{k_{j}}\right)\right| \geq \frac{\lambda}{3}\right] .
$$

By the triangle inequality we have

$$
\left|\widehat{Z}_{t}^{\alpha, c, a}(s)-\widehat{Z}_{t}^{\alpha, c, a}\left(r_{k_{j}}\right)\right| \leq\left|\widehat{Z}_{t}^{\alpha, c, a}(s)-\widehat{Z}_{t}^{\alpha, c, a}\left(r_{i}\right)\right|+\left|\widehat{Z}_{t}^{\alpha, c, a}\left(r_{i}\right)-\widehat{Z}_{t}^{\alpha, c, a}\left(r_{k_{j}}\right)\right| .
$$

Now if $s \in\left[r_{k_{j}}, r_{k_{j+1}}\right]$, then either

$$
\widehat{Z}_{t}^{\alpha, c, a}(s)=\widehat{Z}_{t}^{\alpha, c, a}\left(r_{i}\right) \quad \text { for some } \quad i \in \mathbb{N}
$$

immediately simplifying (4.9), or $\exists i \in \mathbb{N}$ such that

$$
r_{k_{j}} \leq r_{i-1}<s<r_{i} \leq r_{k_{j+1}}
$$

in which case from Lemma 4.5 we have

$$
\begin{aligned}
& \left|\widehat{Z}_{t}^{\alpha, c, a}(s)-\widehat{Z}_{t}^{\alpha, c, a}\left(r_{i}\right)\right| \\
& \leq\left|\widehat{Z}_{t}^{\alpha, c, a}\left(r_{i}\right)-\widehat{Z}_{t}^{\alpha, c, a}\left(r_{i-1}\right)\right|+\left(r_{i}-r_{i-1}\right) t^{1 / 2 \alpha} \\
& =\left|\widehat{Z}_{t}^{\alpha, c, a}\left(r_{i}\right)-\widehat{Z}_{t}^{\alpha, c, a}\left(r_{i-1}\right)\right|+\frac{1}{t^{1 / 2 \alpha}} \\
& \leq\left|\widehat{Z}_{t}^{\alpha, c, a}\left(r_{i}\right)-\widehat{Z}_{t}^{\alpha, c, a}\left(r_{k_{j}}\right)\right|+\left|\widehat{Z}_{t}^{\alpha, c, a}\left(r_{i-1}\right)-\widehat{Z}_{t}^{\alpha, c, a}\left(r_{k_{j}}\right)\right|+\frac{1}{t^{1 / 2 \alpha}} .
\end{aligned}
$$

Therefore, using the inequality (4.9) in conjunction with (4.10) and Lemma 4.6 we have that for $s, r_{i} \in\left[r_{k_{j}}, r_{k_{j+1}}\right]$

$$
\begin{aligned}
& \left|\widehat{Z}_{t}^{\alpha, c, a}(s)-\widehat{Z}_{t}^{\alpha, c, a}\left(r_{k_{j}}\right)\right| \\
\leq & \left|\widehat{Z}_{t}^{\alpha, c, a}\left(r_{i}\right)-\widehat{Z}_{t}^{\alpha, c, a}\left(r_{k_{j}}\right)\right|+\left|\widehat{Z}_{t}^{\alpha, c, a}\left(r_{i-1}\right)-\widehat{Z}_{t}^{\alpha, c, a}\left(r_{k_{j}}\right)\right|+\frac{1}{t^{1 / 2 \alpha}} \\
& +\left|\widehat{Z}_{t}^{\alpha, c, a}\left(r_{i}\right)-\widehat{Z}_{t}^{\alpha, c, a}\left(r_{k_{j}}\right)\right| \\
\leq & 3 \max _{k_{j} \leq i \leq k_{j+1}}\left|\widehat{Z}_{t}^{\alpha, c, a}\left(r_{i}\right)-\widehat{Z}_{t}^{\alpha, c, a}\left(r_{k_{j}}\right)\right|+\frac{1}{t^{1 / 2 \alpha}} \\
\leq & 3\left[\max _{k_{j} \leq i \leq k_{j+1}}\left|Z_{t}^{\alpha, c, a}\left(r_{i}\right)-Z_{t}^{\alpha, c, a}\left(r_{k_{j}}\right)\right|+\frac{7}{3 t^{1 / 2 \alpha}}\right] .
\end{aligned}
$$

Thus

$$
\begin{aligned}
& \mathbb{P}\left[\sup _{r_{k_{j}} \leq s \leq r_{k_{j+1}}}\left|\widehat{Z}_{t}^{\alpha, c, a}(s)-\widehat{Z}_{t}^{\alpha, c, a}\left(r_{k_{j}}\right)\right| \geq \frac{\lambda}{3}\right] \\
\leq & \mathbb{P}\left[\max _{k_{j} \leq i \leq k_{j+1}}\left|Z_{t}^{\alpha, c, a}\left(r_{i}\right)-Z_{t}^{\alpha, c, a}\left(r_{k_{j}}\right)\right|+\frac{7}{3 t^{1 / 2 \alpha}} \geq \frac{\lambda}{9}\right] .
\end{aligned}
$$

Substituting this last inequality into (4.8) gives the statement of the Lemma. 
Now that we have reduced the study of the distribution of the modulus of continuity to that of the maximum fluctuation over our constructed subintervals we can progress by introducing a maximal inequality. In order to do this we use the following known result taken from (마) and paraphrased for use here.

Theorem 4.8. Consider a sequence of random variables $\left\{\xi_{q}\right\}_{q \geq 1}$ and the associated partial sums

$$
S_{u}:=\sum_{q=1}^{u} \xi_{q} \quad S_{0}:=0 .
$$

Let

If

$$
M_{n}:=\max _{1 \leq u \leq n}\left|S_{u}\right|
$$

$$
\mathbb{P}\left[\left|S_{v}-S_{u}\right| \geq \gamma\right] \leq \frac{1}{\gamma^{2}}\left(\sum_{u<l \leq v} b_{l}\right)^{2} \quad 0 \leq u \leq v \leq n
$$

for $\gamma>0$ and some $b_{1}, b_{2}, \ldots, b_{n} \in \mathbb{R}_{+}$, then

$$
\mathbb{P}\left[M_{n} \geq \gamma\right] \leq \frac{\kappa}{\gamma^{2}}\left(\sum_{0<l \leq n} b_{l}\right)^{2}
$$

where $\kappa$ is a constant.

Proof. See (6), Theorem 10.2

\section{Lemma 4.9.}

$$
\mathbb{P}\left[\max _{1 \leq u \leq n}\left|Z_{t}^{\alpha, c, a}\left(r_{k_{j}+u}\right)-Z_{t}^{\alpha, c, a}\left(r_{k_{j}}\right)\right| \geq \gamma\right] \leq \frac{\kappa}{a \gamma^{2}}\left(n t^{-1 / 2 \alpha}\right)^{2},
$$

where $\kappa$ is constant and $\gamma>0$.

Proof. Let

$$
\xi_{q}:=Z_{t}^{\alpha, c, a}\left(r_{k_{j}+q}\right)-Z_{t}^{\alpha, c, a}\left(r_{k_{j}+q-1}\right)
$$

Then

$$
S_{u}:=\sum_{q=1}^{u} \xi_{q}=Z_{t}^{\alpha, c, a}\left(r_{k_{j}+u}\right)-Z_{t}^{\alpha, c, a}\left(r_{k_{j}}\right) \quad S_{0}:=0 .
$$

In this case, for $v, u \in \mathbb{N}$, applying Chebyshev's inequality and using the definition of $f^{\alpha, c, a}(\cdot)$ and the upper bound given at (2.14), we have

$$
\begin{aligned}
\mathbb{P}\left[\left|S_{v}-S_{u}\right| \geq \gamma\right] & =\mathbb{P}\left[\left|Z_{t}^{\alpha, c, a}\left(r_{k_{j}+v}\right)-Z_{t}^{\alpha, c, a}\left(r_{k_{j}+u}\right)\right| \geq \gamma\right] \\
& \leq \frac{f^{\alpha, c, a}\left(\left|r_{k_{j}+v}-r_{k_{j}+u}\right|\right)}{\gamma^{2}} \\
& \leq \frac{(v-u) t^{-1 / \alpha}}{a \gamma^{2}} \\
& \leq \frac{\left[(v-u) t^{-1 / 2 \alpha}\right]^{2}}{a \gamma^{2}} .
\end{aligned}
$$

Thus we can take $b_{l}=\frac{t^{-1 / 2 \alpha}}{a}$ for $l=1,2, \ldots, n$ and apply Theorem 4.8 which gives the maximal inequality of the Lemma. 
Concluding the proof of Proposition 4.4 is now straightforward.

Proof of Proposition 4.4. Taking $n=k_{j+1}-k_{j}$ in the statement of Lemma 4.9 gives

$$
\begin{aligned}
\mathbb{P}\left[\max _{k_{j} \leq i \leq k_{j+1}}\left|Z_{t}\left(r_{i}\right)-Z_{t}\left(r_{k_{j}}\right)\right| \geq \gamma\right] & \leq \frac{\kappa}{a \gamma^{2}}\left(r_{k_{j+1}}-r_{k_{j}}\right)^{2} \\
& \leq \frac{\kappa}{a \gamma^{2}}\left(\delta+t^{-1 / \alpha}\right)^{2}
\end{aligned}
$$

Substituting this last inequality with $\gamma=\left(\frac{\lambda}{9}-\frac{7}{3 t^{1 / 2 \alpha}}\right)$ (which is strictly positive for sufficiently large $t$ ) into the inequality given by Lemma 4.7 gives

$$
\mathbb{P}\left[w\left(Z_{t}^{\alpha, c, a}, \delta\right) \geq \lambda\right] \leq \frac{81 m \kappa}{a} \frac{\left(\delta t^{1 / 2 \alpha}+t^{-1 / 2 \alpha}\right)^{2}}{\left(\lambda t^{1 / 2 \alpha}-21\right)^{2}} .
$$

On solving the appropriate quadratic equation we find that we can make this upper bound less than $\epsilon$ by choosing $\delta$ from

$$
\left(-t^{-1 / 2 \alpha}-c_{\epsilon}\left(\lambda-21 t^{-1 / 2 \alpha}\right),-t^{-1 / 2 \alpha}+c_{\epsilon}\left(\lambda-21 t^{-1 / 2 \alpha}\right)\right) \bigcap(0,1)
$$

where $c_{\epsilon}=\sqrt{\frac{a \epsilon}{81 m \kappa}}$. Since this interval is non-empty for sufficiently large $t$ this completes the proof.

\subsection{Properties of the limiting process $G^{\alpha, c, a}(s)$}

We have constructed a family $\left\{\left(G^{\alpha, c, a}(s), s \in[0, \infty)\right), \alpha \in(0,2], c>0, a \in \mathbb{R}_{+}\right\}$of centered, continuous, real-valued Gaussian processes with the inherited covariance structure

$$
\operatorname{Cov}\left(G^{\alpha, c, a}(s), G^{\alpha, c, a}(r)\right)=\frac{1}{2}\left(f^{\alpha, c, a}(s)+f^{\alpha, c, a}(r)-f^{\alpha, c, a}(|s-r|)\right) .
$$

It is clear that the processes are recurrent for $1<\alpha \leq 2$ as the number variance saturates and the stationary distribution is normal with mean zero and variance $\kappa_{s a t}(\alpha, c, a, 1)$. We are able to deduce further properties of these limit processes by using our earlier results on the number variance for the systems of symmetric $\alpha$-stable processes from which they are constructed.

Propostition 4.10. $G^{\alpha, c, a}(s)$ is non-Markovian.

Proof. Recall (see e.g. (18), Chapter 13) that a Gaussian process with indexing set $T \subset \mathbb{R}$ and covariance function $\rho: T \mapsto \mathbb{R}$ is Markov if and only if

$$
\rho(s, u)=\frac{\rho(s, r) \rho(r, u)}{\rho(r, r)} \quad \forall s, u, r \in T .
$$

It is clear that this relationship does not hold in general for the covariance function (4.11).

From the results of previous sections, since $f^{\alpha, c, a}(s)=V_{1}^{\alpha, c, a}[s]$, we know that

$$
f^{\alpha, c, a}(s) \rightarrow\left(1-e^{-2 c}\right) \frac{s}{a} \quad \text { as } \alpha \rightarrow 0
$$


and

$$
f^{\alpha, c, a}(s) \stackrel{s \rightarrow 0}{\sim} k_{\alpha, c, a} s
$$

Therefore, $G^{\alpha, c, a}$ appears to start out, for small "time" as a scaled Brownian motion and as $\alpha \rightarrow 0$ this initial Brownian character prevails for longer. We capture this more precisely in the following easily verified proposition.

Propostition 4.11. 1. $\left\{G^{\alpha, c, a}(s): s \in[0,1]\right\}$ converges weakly to a Brownian motion $\left\{B\left(\frac{1-e^{-2 c}}{a} s\right): s \in[0,1]\right\}$ as $\alpha \rightarrow 0$.

2. Let $G_{\epsilon}^{\alpha, c, a}(s)=\epsilon^{-1 / 2} G^{\alpha, c, a}(\epsilon s)$. Then $\left\{G_{\epsilon}^{\alpha, c, a}(s): s \in[0,1]\right\}$ converges weakly to a Brownian motion $\left\{B\left(\frac{s}{a}\right): s \in[0,1]\right\}$ as $\epsilon \rightarrow 0$.

Remark 4.12. The covariance structure of $G^{\alpha, c, a}$ is similar to that of a Brownian bridge. Recall that the standard Brownian bridge $\left(B^{b r}(s), s \in[0, a]\right)$ of length $a$, is a centered Gaussian process with covariance structure

$$
\operatorname{Cov}\left(B^{b r}(s), B^{b r}(r)\right)=s \wedge r-\frac{s r}{a}
$$

and arises as a weak limit of many empirical processes. In particular, it may be obtained from the appropriately scaled counting functions of a Poisson process on $\mathbb{R}$ (see e.g. (18)). We can re-write the covariance (4.1) in the alternative form

$$
\operatorname{Cov}\left(G^{\alpha, c, a}(s), G^{\alpha, c, a}(r)\right)=\frac{s \wedge r}{a}-\int_{0}^{\frac{r}{a}} \int_{0}^{\frac{s}{a}} p_{2 / a^{\alpha}}(y, z) \mathrm{d} y \mathrm{~d} z,
$$

but we see that a precise match would require $p_{2 / a^{\alpha}}(y, z)=1$.

Propostition 4.13. The process $G^{\alpha, c, a}$ has stationary increments which are negatively correlated.

Proof. It is straightforward to see that the increments have zero mean and that for any $s, r \in$ $[0, \infty)$

$$
\operatorname{Var}\left(G^{\alpha, c, a}(s)-G^{\alpha, c, a}(r)\right)=f^{\alpha, c, a}(|s-r|) .
$$

In addition, for $u \geq 0$ we have

$$
\begin{aligned}
& \operatorname{Cov}\left(G^{\alpha, c, a}(s)-G^{\alpha, c, a}(0), G^{\alpha, c, a}(r+s+u)-G^{\alpha, c, a}(s+u)\right) \\
= & \frac{1}{2}\left(f^{\alpha, c, a}(s+r+u)-f^{\alpha, c, a}(r+u)-\left[f^{\alpha, c, a}(s+u)-f^{\alpha, c, a}(u)\right]\right) .
\end{aligned}
$$

Since $f^{\alpha, c, a}$ is a concave function

$$
f^{\alpha, c, a}(s+r+u)-f^{\alpha, c, a}(r+u) \leq f^{\alpha, c, a}(s+u)-f^{\alpha, c, a}(u),
$$

for all $s, r, u \in[0, \infty)$ so it follows that this covariance is non-positive.

Propostition 4.14. $G^{\alpha, c, a}$ is not in general self-similar. For any constant $b \in \mathbb{R}$ we have the relationship

$$
G^{\alpha, c, a}(b s) \stackrel{\text { dist }}{=} b^{1 / 2} G^{\alpha, \frac{c}{b^{\alpha}}, a}(s)
$$


Proof. Both sides of the proposed equation have zero mean and a Gaussian distribution. It is clear from the expression given for $f^{\alpha, c, a}$ at (3.7) that the variances/covariances also agree.

Propostition 4.15. $G^{\alpha, c, a}$ is a long memory (or long range dependent) process in the sense that the covariance between increments decays as a power law as the separation between them is increased. More precisely, for $\alpha \in(0,2)$ we have

$$
\operatorname{Cov}\left(G^{\alpha, c, a}(s)-G^{\alpha, c, a}(0), G^{\alpha, c, a}(r+s+u)-G^{\alpha, c, a}(s+u)\right) \stackrel{u \rightarrow \infty}{\sim} k u^{-(\alpha+1)},
$$

where $k$ is a constant depending on $\alpha, c, a, s$ and $r$.

Proof. The covariance in question is expressed in terms of the function $f^{\alpha, c, a}$ at (4.12). Note from (2.10) that we already know the asymptotic behaviour of the individual components of this expression. Applying l'Hopital's rule twice in succession yields the given power law.

We have already mentioned a similarity between the covariance structure of $G^{\alpha, c, a}$ and that of Brownian motion. More generally we can draw parallels between our limiting process and fractional Brownian motion. Recall (see for example (21)) that a fractional Brownian motion $\left(W_{H}(s), s \geq 0\right)$ with Hurst parameter $H \in(0,1)$ is a centered, self-similar Gaussian process with covariance function

$$
\operatorname{Cov}\left(W_{H}(s), W_{H}(r)\right)=\frac{1}{2}\left(s^{2 H}+r^{2 H}-|s-r|^{2 H}\right),
$$

The case $H=\frac{1}{2}$ corresponds to a standard Brownian motion.

Note the resemblance between the form of the covariance functions (4.11) and (4.13). Heuristically, we can deduce that $f^{\alpha, c, a}(s)$ may be approximated by a function of the form $\kappa_{\alpha, c, a} s^{2 H_{\alpha, c, a}(s)}$ where $H_{\alpha, c, a}:[0, \infty) \mapsto\left[0, \frac{1}{2}\right]$ is a monotonically decreasing function with initial value $H_{\alpha, c, a}(0)=\frac{1}{2}$. Thus loosely speaking $G^{\alpha, c, a}$ can be viewed as a type of fractional Brownian motion with time varying Hurst parameter. In particular, the long range dependence property of Proposition 4.15 may be compared to the analogous statement for fractional Brownian motion:

$$
\operatorname{Cov}\left(W_{H}(s)-W_{H}(0), W_{H}(r+s+u)-W_{H}(s+u)\right) \stackrel{u \rightarrow \infty}{\sim} k u^{2 H-2},
$$

where $k$ is a constant depending on $H, s$ and $r$. We make the link between the process $G^{\alpha, c, a}$ and fractional Brownian motion precise with the following statement.

Propostition 4.16. For $\alpha \in(0,1)$ let

$$
\widetilde{G}_{b}^{\alpha, c, a}(s):=\frac{G^{\alpha, c, a}(b s)}{\sqrt{b^{1-\alpha}}}, \quad s, b \in[0, \infty) .
$$

Then

$$
\left\{\widetilde{G}_{b}^{\alpha, c, a}(s): s \in[0, \infty)\right\} \Rightarrow\left\{k_{\alpha, c, a}^{1 / 2} W_{\frac{1-\alpha}{2}}(s): s \in[0, \infty)\right\} \quad \text { as } b \rightarrow \infty
$$

where $k_{\alpha, c, a}=\frac{4 c}{a \pi} \Gamma(\alpha-1) \sin (-\alpha \pi / 2)$. 
Proof. From (3.7), by applying a Taylor expansion we deduce that

$$
\begin{aligned}
\frac{f^{\alpha, c, a}(b s)}{b^{1-\alpha}}= & s^{1-\alpha} \frac{8 c}{a \pi} \int_{0}^{\infty} \frac{\sin ^{2}(u / 2)}{u^{2-\alpha}} \mathrm{d} u \\
& +\frac{4}{a \pi} \int_{0}^{\infty} \sin ^{2}(u / 2) \sum_{j=2}^{\infty} \frac{(-1)^{j+1}}{j !}(2 c)^{j} s^{1-2 j \alpha} \frac{u^{j \alpha-2}}{b^{j \alpha-\alpha}} \mathrm{d} u .
\end{aligned}
$$

Now by the Dominated Convergence Theorem,

$$
\begin{aligned}
& \lim _{b \rightarrow \infty} \frac{4}{a \pi} \int_{0}^{\infty} \underbrace{\sin ^{2}(u / 2) \sum_{j=2}^{\infty} \frac{(-1)^{j+1}}{j !}(2 c)^{j} s^{1-2 j \alpha} \frac{u^{j \alpha-2}}{b^{j \alpha-\alpha}}}_{h_{b}(u)} \mathrm{d} u \\
& \quad=\frac{4}{a \pi} \int_{0}^{\infty} \sin ^{2}(u / 2) \sum_{j=2}^{\infty} \lim _{b \rightarrow \infty} \frac{(-1)^{j+1}}{j !}(2 c)^{j} s^{1-2 j \alpha} \frac{u^{j \alpha-2}}{b^{j \alpha-\alpha}} \mathrm{d} u, \\
& \quad=0
\end{aligned}
$$

since, setting $M(u)=\sin ^{2}(u / 2)\left(1-\exp \left(-2 c(u / s)^{\alpha}\right)\right) / u^{2}+\sin ^{2}(u / 2) / u^{2-\alpha}$, we have a positive integrable function such that $\left|h_{b}(u)\right| \leq M(u)$ for all $b \in \mathbb{R}$. This implies that

$$
\lim _{b \rightarrow \infty} \frac{f^{\alpha, c, a}(b s)}{b^{1-\alpha}}=k_{\alpha, c, a} s^{1-\alpha}
$$

which allows us to conclude

$$
\begin{aligned}
\operatorname{Cov}\left(\widetilde{G}_{b}^{\alpha, c, a}(r), \widetilde{G}_{b}^{\alpha, c, a}(s)\right) & =b^{\alpha-1} \operatorname{Cov}\left(G^{\alpha, c, a}(b r), G^{\alpha, c, a}(b s)\right) \\
& \stackrel{b \rightarrow \infty}{\rightarrow} \frac{k_{\alpha, c, a}}{2}\left(s^{1-\alpha}+r^{1-\alpha}+|s-r|^{1-\alpha}\right) \\
& =\operatorname{Cov}\left(k_{\alpha, c, a}^{1 / 2} W_{\frac{1-\alpha}{2}}(s), k_{\alpha, c, a}^{1 / 2} W_{\frac{1-\alpha}{2}}(r)\right) .
\end{aligned}
$$

The processes are Gaussian therefore the convergence of finite dimensional distributions is implied by the convergence of the covariance functions and tightness follows easily from, for example, (18) Corollary 16.9 and well known expressions for the even moments.

Remark 4.17. We mention that various other long-range dependent Gaussian processes have recently been found to arise from the fluctuations of spatially distributed particle systems, see (12) and references within. Notably, in this context, the spatial particle configurations of infinite systems of symmetric $\alpha$-stable processes started from a Poisson process on $\mathbb{R}$ have been considered. In these cases, fractional Brownian motion with Hurst parameter $H=1-\frac{1}{2 \alpha}, \alpha \in(1,2]$ was obtained as a scaling limit of the occupation time process (essentially scaling the counting function in time rather than in time and space as in this paper).

Remark 4.18. It seems natural to ask whether, in the same fashion as we created $G^{\alpha, c, a}$, similar limiting processes could be constructed from Johansson's systems of non-colliding Brownian motions. Unfortunately, the formula for the averaged number variance given for these processes in (16) does not scale in time and space in the same convenient way as $V_{t}^{\alpha, c, a}[L]$ in this case. However, as noted by Johansson, letting $t \rightarrow \infty$ in his model, one obtains the sine kernel determinantal process, from which a limiting Gaussian process (parameterized and scaled in a completely different way) was constructed in (26). 


\section{References}

[1] R. Aurich and F. Steiner. Periodic-orbit theory of the number variance $\Sigma^{2}(L)$ of strongly chaotic systems. Phys. D, 82(3):266-287, 1995. MR1326559

[2] A. D. Barbour and P. Hall. On the rate of Poisson convergence. Math. Proc. Cambridge Philos. Soc., 95(3):473-480, 1984. MR0755837

[3] M. V. Berry. The Bakerian lecture, 1987. Quantum chaology. Proc. Roy. Soc. London Ser. A, 413(1844):183-198, 1987. MR0909277

[4] M. V. Berry. Semiclassical formula for the number variance of the Riemann zeros. Nonlinearity, 1(3):399-407, 1988. MR0955621.

[5] M.V. Berry and J.P. Keating. The Riemann zeros and eigenvalue asymptotics. SIAM review, 41:236-266, 1999. MR1684543

[6] P. Billingsley. Convergence of probability measures. Wiley Series in Probability and Statistics: Probability and Statistics. John Wiley \& Sons Inc., New York, 1999. MR1700749

[7] O. Bohigas, M.J. Giannoni, and C. Schmidt. Characterizaton of chaotic quantum spectra and universality of level fluctuation laws. Phys.Rev.Lett, 52:1-4, 1984. MR0730191]

[8] O. Costin and J. Lebowitz. Gaussian fluctuations in random matrices. Phys.Rev.Lett, 75:69-72, 1995.

[9] J. L. Doob. Stochastic processes. John Wiley \& Sons Inc., New York, 1953. MR0058896

[10] W. Feller. An introduction to probability theory and its applications. Vol. II. Second edition. John Wiley \& Sons Inc., New York, 1971. MR0270403

[11] S. Goldstein, J. L. Lebowitz, and E. R. Speer. Large deviations for a point process of bounded variability. Markov Process. Related Fields, 12(2):235-256, 2006. [MR2249630

[12] L. G. Gorostiza, R. Navarro, and E. R. Rodrigues. Some long-range dependence processes arising from fluctuations of particle systems. Acta Appl. Math., 86(3):285-308, 2005. MR2136367

[13] T. Guhr and A. Müller-Groeling. Spectral correlations in the crossover between GUE and Poisson regularity: on the identification of scales. J. Math. Phys., 38(4):1870-1887, 1997. MR1450904

[14] Valerie Isham and Mark Westcott. A self-correcting point process. Stochastic Process. Appl., 8(3):335-347, 1978/79. MR0535308

[15] K. Johansson. Universality of the local spacing distribution in certain ensembles of Hermitian Wigner matrices. Comm. Math. Phys., 215(3):683-705, 2001. MR1810949

[16] K. Johansson. Determinantal processes with number variance saturation. Comm. Math. Phys., 252(1-3):111-148, 2004. MR2103906 
[17] Liza Jones and Neil O'Connell. Weyl chambers, symmetric spaces and number variance saturation. ALEA Lat. Am. J. Probab. Math. Stat., 2:91-118 (electronic), 2006. MR2249664]

[18] O. Kallenberg. Foundations of modern probability. Probability and its Applications (New York). Springer-Verlag, New York, 2002. MR1876169

[19] T. Lewis and L. J. Govier. Some properties of counts of events for certain types of point process. J. Roy. Statist. Soc. Ser. B, 26:325-337, 1964. MR0178516

[20] W. Luo and P. Sarnak. Number variance for arithmetic hyperbolic surfaces. Comm. Math. Phys., 161(2):419-432, 1994. MR1266491

[21] B. B. Mandelbrot and J. W. Van Ness. Fractional Brownian motions, fractional noises and applications. SIAM Rev., 10:422-437, 1968. MR0242239

[22] M. L. Mehta. Random matrices, volume 142 of Pure and Applied Mathematics (Amsterdam). Elsevier/Academic Press, Amsterdam, 2004. MR2129906

[23] G. Samorodnitsky and M. S. Taqqu. Stable non-Gaussian random processes. Stochastic Modeling. Chapman \& Hall, New York, 1994. MR1280932

[24] K. Sato. Lévy processes and infinitely divisible distributions, volume 68 of Cambridge Studies in Advanced Mathematics. Cambridge University Press, Cambridge, 1999. MR1739520

[25] D. N. Shanbhag and M. Sreehari. On certain self-decomposable distributions. Z. Wahrscheinlichkeitstheorie und Verw. Gebiete, 38(3):217-222, 1977. MR0436267.

[26] A. Soshnikov. Level spacings distribution for large random matrices: Gaussian fluctuations. Ann. of Math. (2), 148(2):573-617, 1998. MR1668559

[27] A. Soshnikov. Determinantal random point fields. Uspekhi Mat. Nauk, 55(5(335)):107-160, 2000. MR1799012

[28] A. Soshnikov. Gaussian fluctuation for the number of particles in Airy, Bessel, sine, and other determinantal random point fields. J. Statist. Phys., 100(3-4):491-522, 2000. MR1788476 